\title{
Education and public outreach of the Pierre Auger Observatory
}

\author{
Charles Timmermans $^{* a}$ for the Pierre Auger Collaboration ${ }^{b}$ \\ ${ }^{a}$ Nikhef and Radboud University, Nijmegen, the Netherlands \\ ${ }^{b}$ Observatorio Pierre Auger, Av. San Martín Norte 304, 5613 Malargüe, Argentina \\ E-mail: auger_spokespersons@fnal.gov \\ Full author list: : http://www.auger.org/archive/authors_icrc_2017.html
}

\begin{abstract}
The scale and scope of the physics studied at the Pierre Auger Observatory continue to offer significant opportunities for original outreach work. Education, outreach and public relations of the Auger Collaboration are coordinated in a separate task whose goals are to encourage and support a wide range of education and outreach efforts that link schools and the public with the Auger scientists and the science of cosmic rays, particle physics, and associated technologies. This paper focuses on the impact of the Collaboration in Mendoza Province, Argentina. The Auger Visitor Center in Malargüe has hosted over 110,000 visitors since 2001, and a sixth Collaborationsponsored science fair was held on the Observatory campus in November 2016. Numerous online resources, video documentaries, and animations of extensive air showers have been created for wide public release. Increasingly, collaborators draw on these resources to develop Auger-related displays and outreach events at their institutions and in public settings to disseminate the science and successes of the Observatory worldwide. The presentation will also highlight the impact of the recently renovated Visitor Center, configured now to allow self-guided tours, which has increased the visitor count over the last year and provided a boost to local outreach.
\end{abstract}

35th International Cosmic Ray Conference - ICRC2017

10-20 July, 2017

Bexco, Busan, Korea

${ }^{*}$ Speaker. 


\section{Introduction}

Education and public outreach (EPO) have been an integral part of the Pierre Auger Observatory since its inception. The collaboration's EPO activities are organized in a separate Education and Outreach Task that was established in 1997. With the Observatory headquarters located in the remote city of Malargüe, population 28,000, early outreach activities, which included public talks, visits to schools, and courses for science teachers and students, were aimed at familiarizing the local population with the science of the Observatory and the presence of the large Collaboration of international scientists in the isolated communities and countryside of Mendoza Province. As an example of the Observatory's integration into local traditions, the Collaboration has participated in the annual Malargüe Day parade since 2001 with collaborators marching behind a large Auger banner (see Fig. 1). Close contact with the community fosters a sense of ownership and being a part of our scientific mission. The Observatory's EPO efforts have been documented in previous ICRC contributions [1, 2,3]. Fifteen years after opening to the public, the Auger Visitor Center has been modernized, and, in parallel, web-based outreach efforts are intensified.

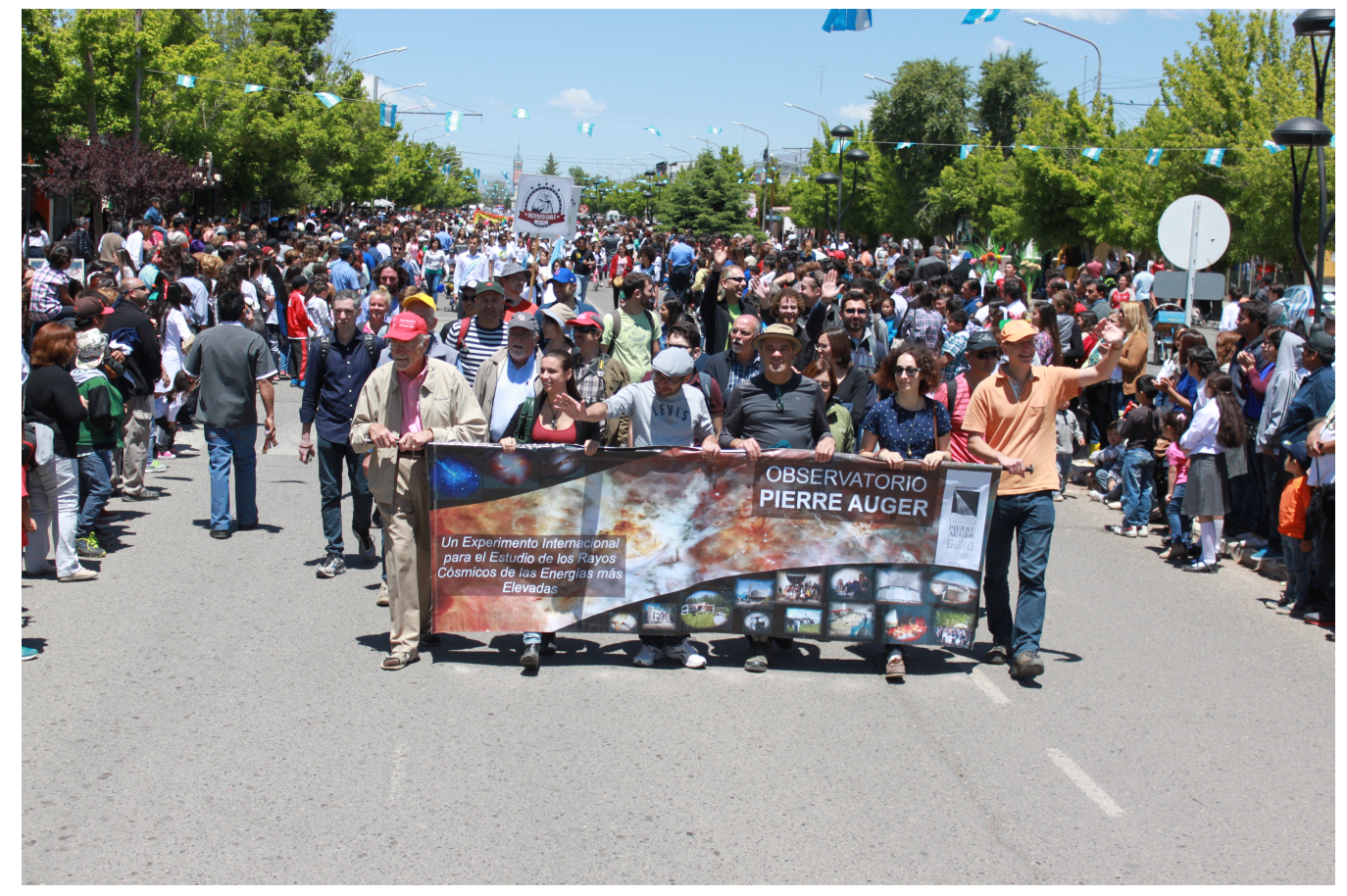

Figure 1: Auger collaborators participating in the November 2016 Malargüe Day parade.

\section{The Auger Visitor Center in Malargüe}

The Auger Visitor Center (VC), located in the central office complex in Malarguie, continues to be a popular attraction. The VC is managed by a small staff led by an Observatory employee; they share the task of giving presentations and tours to visitors and school groups. Through May, 2017, the VC has hosted 110,920 visitors. Fig. 2 shows the integrated number of visitors since 
Nov. 2001. The noticeable increase of visitors since 2008 occurred after the opening of a nearby planetarium [4], which is operated independently of the Observatory, in August of that year. A recent increase in the number of visitors occurred in 2016 after the opening of the modernized Visitor Center. An upgrade of the Observatory's detector systems, currently in progress, provided
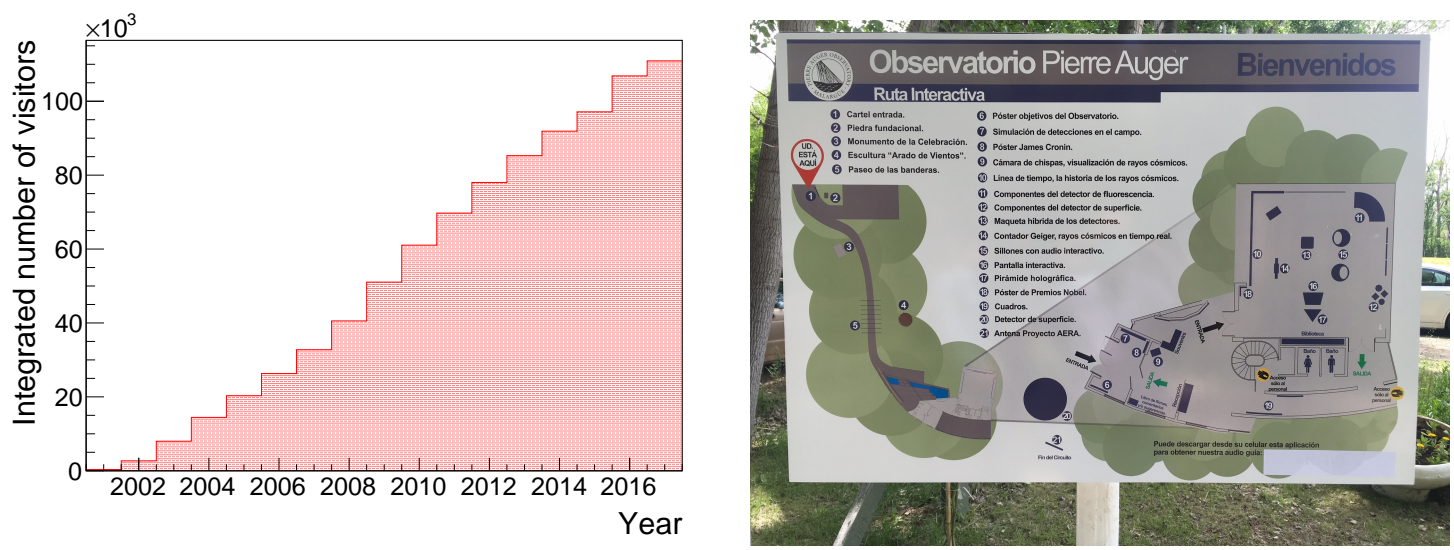

Figure 2: Left: The integrated number of visitors logged at the Auger Visitor Center since 2001. Right: The self guided tour starts outside in the garden of the Observatory.

an incentive to modernize the $\mathrm{VC}$, and thereby re-inviting people who have seen the Observatory in the past to relive this experience through a self-guided tour. As shown in Fig. 2, this self-guided tour starts in the beautiful gardens of the Observatory where several monuments commemorate the inauguration of the Observatory and highlight the international character thereof. Furthermore, actual detectors are on display. In the VC, located inside the James Cronin office building, the tour continues. Audio and video displays explain the effect of cosmic rays in the atmosphere, methods
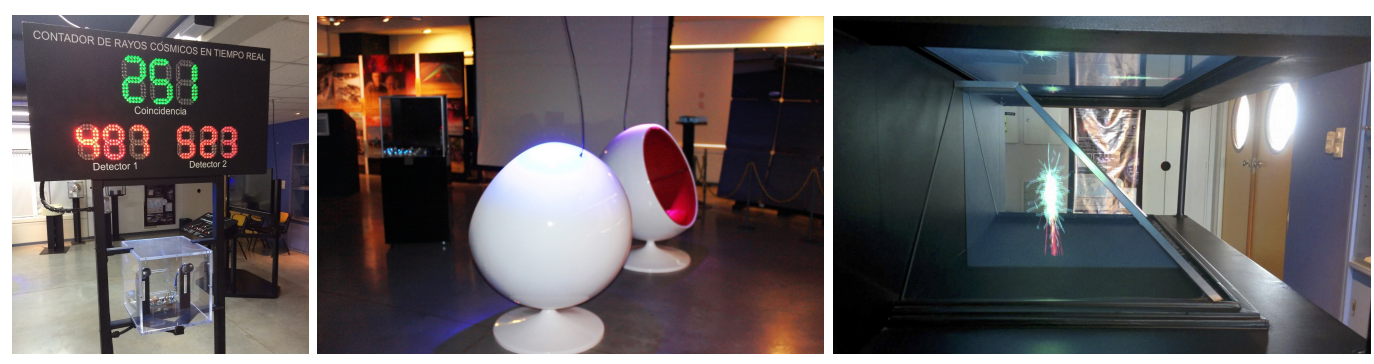

Figure 3: Different elements of the upgraded Auger Visitor Center in Malargüe. From left to right: Geiger Müller counters provide live information on the number of muons, pods describe different features of the Observatory, a 'holographic' display of an air shower shows the development of air showers to the public.

to measure cosmic rays, and the workings of the Observatory. Several objects and 3D models help to understand the different topics, as shown in Fig. 3. These updates to the VC demonstrate a genuine interest of the Collaboration to communicate with the people from Mendoza Province and beyond about the science and tools of the Observatory. In addition, the VC still allows for lectures to school audiences, as shown in Fig. 4. 


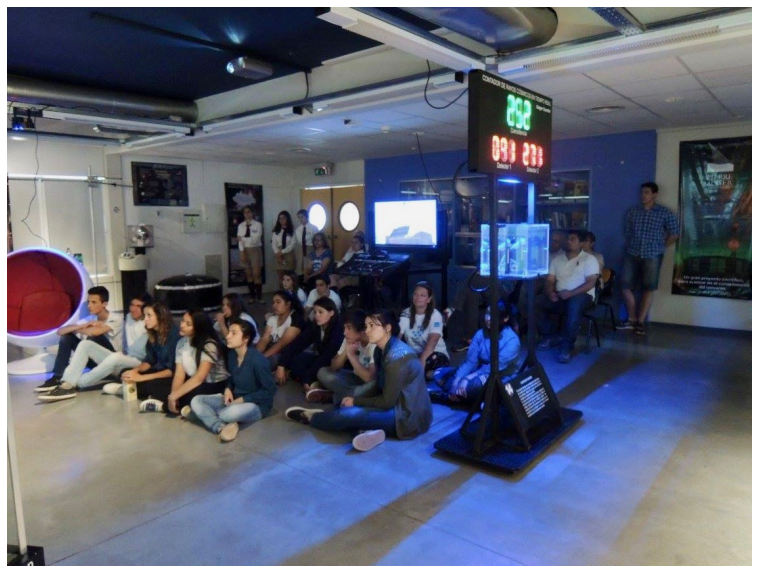

Figure 4: High school students listening to a presentation on the working of the Observatory inside the modernized VC.

\section{The 2016 Auger Science Fair}

The Observatory hosted its sixth biannual Science Fair in the Assembly Building on November 17-19, 2016, as shown in Fig. 5. Twenty-four student teams from all over Mendoza Province, with ages ranging from primary school through high school, presented research projects in the areas of natural science, exact science, and technology. More than 20 Auger collaborators, from different nationalities, and a few invitees served as judges for the student projects. Prizes were awarded to the top teams in several categories in the closing ceremony on November 19. The November 2016 Science Fair owes its success to the Observatory staff, the collaborators who served as judges, the Municipality of Malargüe, the participating teachers and students, and special mention goes to the lead local organizers: Miguel Herrera, Fabian Amaya, and Alicia Piastrellini.
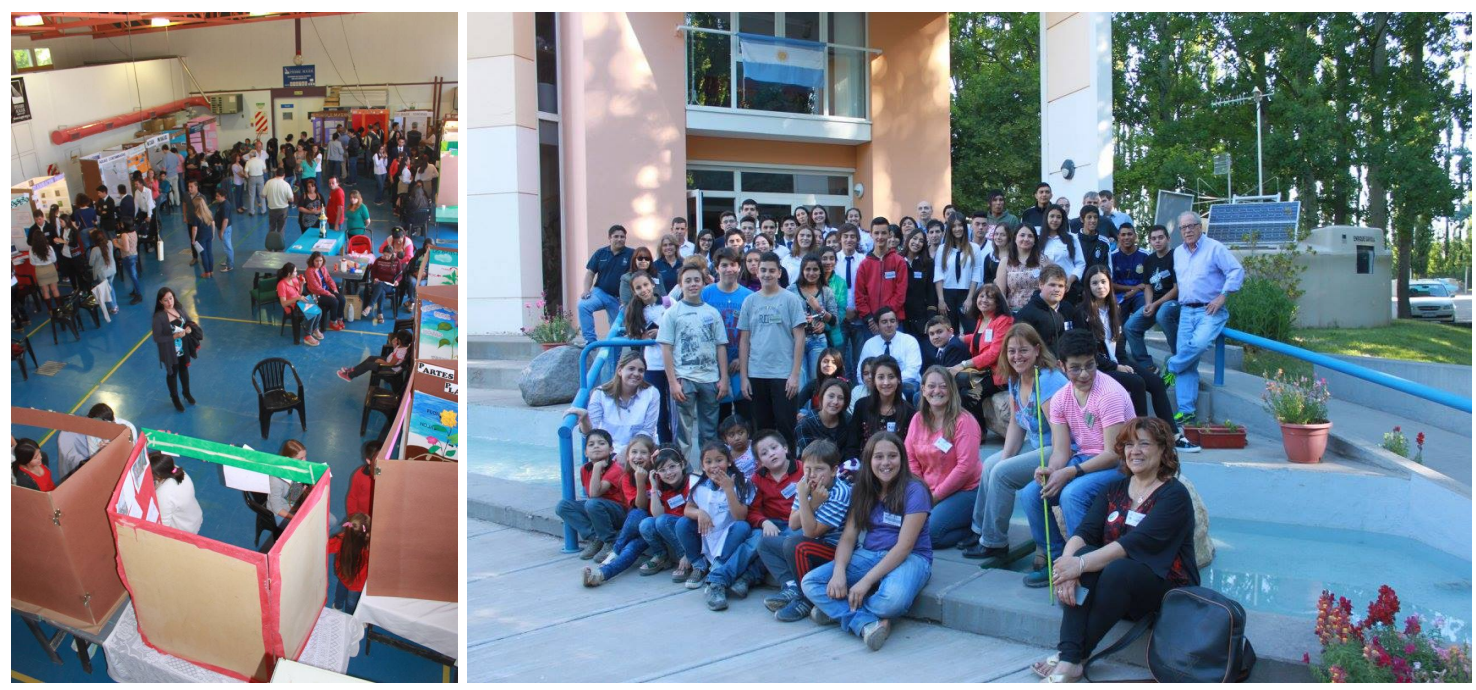

Figure 5: Left: An impression of the 2016 Science Fair. Right: The participants of the Fair. 


\section{The Online Observatory}

The Auger public event display allows the general public to see what information is recorded from an incoming air shower, and to grasp the steps involved in obtaining information on the incoming cosmic ray creating this air shower. The Collaboration has committed to providing $10 \%$ of its data to the general public. The target group for outreach purposes includes even university students for whom statistical tools and analysis packages such as VISPA [6, 7, 8, 9] (see Fig. 6) are available to handle substantial amounts of data. At the same time, high-school students are able to make online

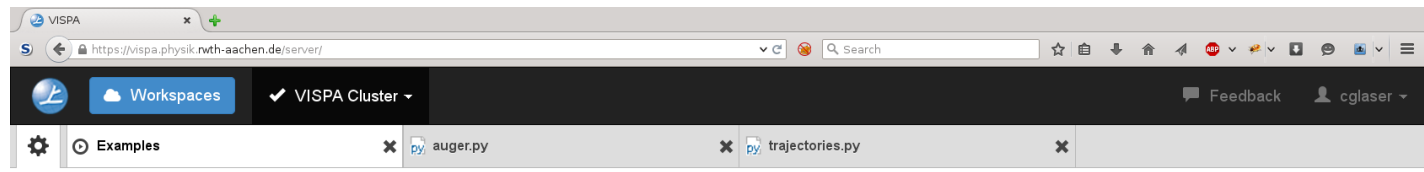

Astroparticle Examples

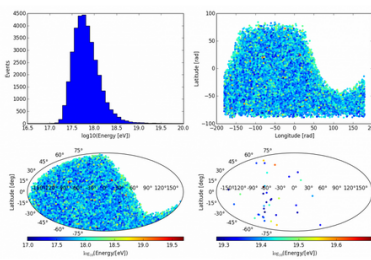

Auger example In this example, you can use the public data set ( $1 \%$ of all events) of the Pierre Auger Observatory to study the energy distribution and to plot the arrival direction of cosmic rays.

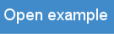

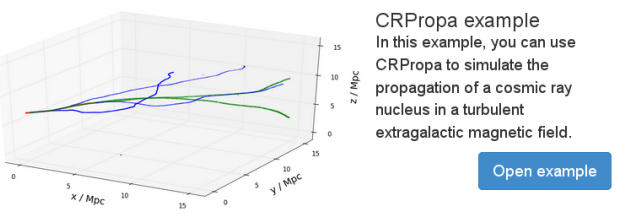

Figure 6: Example of the web-based VISPA public data analysis package. Left: Examples using the Auger public data set. Right: Simulations using the CRPropa package [10].

selections on the events they would like to see or use for their own purposes thereby reducing the data volume while increasing the number of interesting events substantially with respect to when using the more basic tools they are used to.

The self-guided tour described above triggered a presence of the Observatory on the izi.travel website, thus providing the option of a virtual tour of the Observatory [11]. In addition, the Collaboration communicates with the general public through its Facebook page [12], that is used by science fair participants as well as the general public. In addition to providing information about the status of the Observatory, the Collaboration also writes summaries of recent scientific achievements on its main homepage [13] (see Fig. 7). All information and tools combined provide a good overview about the detectors and achievements of the Collaboration, as well as an invitation to the general public to use the data provided by the Collaboration for their own inquiry.

\section{Conclusions}

The Pierre Auger Observatory continues to provide unique education and outreach opportunities which expose people of all ages to the excitement of astroparticle physics. Its Visitor Center and Science Fairs have great local impact near Malargüe, while collaborators from around the world ensure that the Observatory's science and successes have international reach. The upgrade of the Observatory has provided an excellent opportunity to modernize the Visitor Center and strengthen the relations between the Collaboration and the local community. 


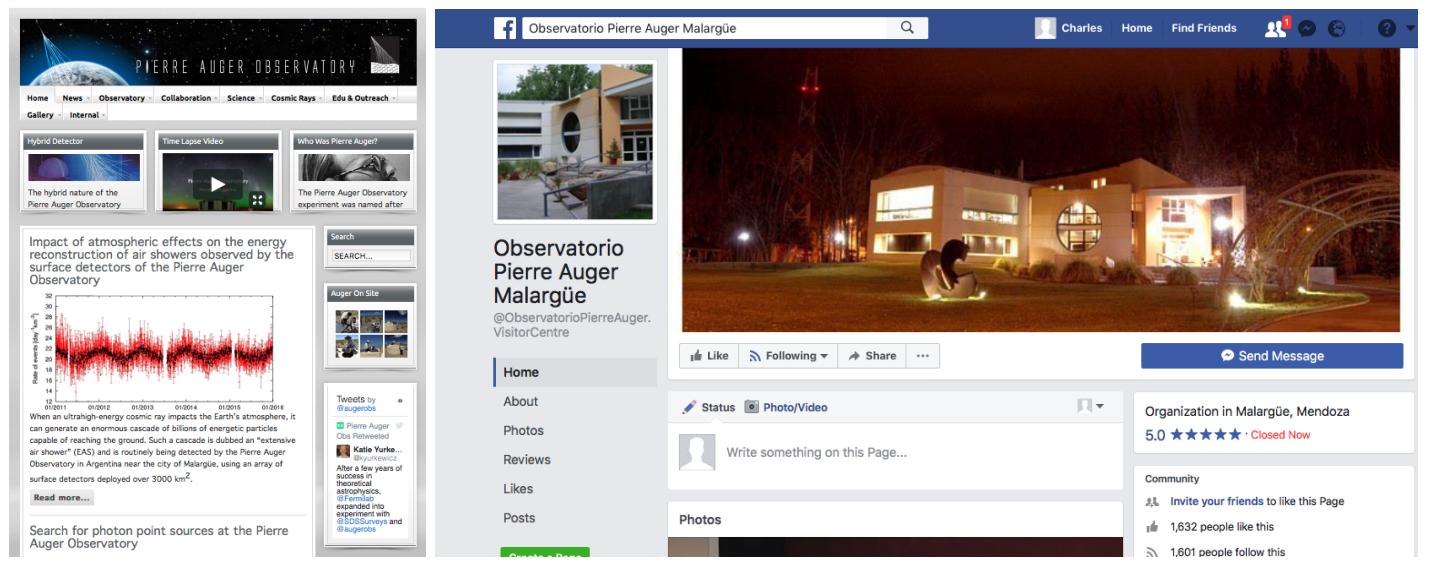

Figure 7: Left: The home page of the Pierre Auger Collaboration. Right: The Facebook page of the Pierre Auger Collaboration.

The improved presence of the Collaboration on the internet allows people across the globe to visit the Observatory, interact with scientists and learn about the exciting new knowledge that scientists have extracted from the data obtained by the Observatory.

\section{References}

[1] Pierre Auger Collaboration, C. Timmermans et al., Education, Outreach and Public Relations of the Pierre Auger Observatory, Proc. 34th ICRC 2015, The Hague, The Netherlands, PoS(ICRC2015)587.

[2] Pierre Auger Collaboration, G. Snow et al., Education and Outreach Activities of the Pierre Auger Observatory, Proc. 33rd ICRC, Rio de Janeiro, Brazil, 2 (2013) 116 [arXiv:1307.5059].

[3] Pierre Auger Collaboration, G. Snow et al., Education and Public Outreach of the Pierre Auger Observatory, Proc. 32nd ICRC, Beijing, China, 2 (2011) 190 [arXiv:1107.4806].

[4] See http://www.planetariomalargue.com.ar

[5] See https://www.auger.org/index.php/edu-outreach/event-display

[6] See http://vispa.physik.rwth-aachen.de

[7] H. P. Bretz et al., A Development Environment for Visual Physics Analysis, JINST 7 (2012) T08005, [arXiv:1205.4912].

[8] M. Erdmann et al., A field study of data analysis exercises in a bachelor physics course using the internet platform VISPA, Eur. J. Phys. 35 (2014) 035018, DOI: 10.1088/0143-0807/35/3/035018, [arXiv:1402.2836]

[9] D. van Asseldonk et al., The VISPA internet platform for outreach, education and scientific research in various experiments, to appear in 21st International Conference on Computing in High Energy and Nuclear Physics (CHEP2015), Okinawa, Japan, (2015).

[10] K. H. Kampert et al., CRPropa 2.0 - a Public Framework for Propagating High Energy Nuclei, Secondary Gamma Rays and Neutrinos, (2012) [arXiv:1206.3132].

[11] See https://izi.travel/en/6095-pierre-auger-observatory/en\#db48-pierre-auger-observatory/en 
[12] See https://www.facebook.com/ObservatorioPierreAuger.VisitorCentre/

[13] See https://www.auger.org 